with small bowel sarcoidosis, ${ }^{3}$ the association between intestinal sarcoid and villous atrophy is unknown.

Crohn's disease and sarcoidosis have common systemic manifestations, and the most frequently occurring cutaneous lesion to be associated with both conditions is erythema nodosum. However, cutaneous granulomas do occur in Crohn's disease, though rarely in areas which are not a direct extension from the gastrointestinal tract or of skin apposition. ${ }^{7}$ Respiratory tract disease, in the form of bronchial granulomas, has been reported in Crohn's disease but in the affected patients there was no other evidence of lung disease. ${ }^{8}$

Coexistent Crohn's disease and sarcoidosis has been reported in a few patients, ${ }^{9}$ and because of their histological and immunological similarities it has been postulated that they may represent an overlapping syndrome of granuloma formation due to a generalised host response to various aetiological agents. However, the existence of non-progressive small bowel disease over two decades, together with a positive Kveim test, is much more compatible with sarcoidosis than Crohn's disease. ${ }^{10}$

Given the protean nature of sarcoidosis, the generally benign course, and the occurrence of asymptomatic lesions it could be that intestinal disease is commoner than previously thought. Patients with sarcoidosis who present with persistent diarrhoea, malabsorption, or folate deficiency should be investigated for intestinal sarcoidosis, and the diagnosis should be considered in patients with small bowel pathology who later develop manifestations of systemic sarcoidosis.

1 Miyamoto C, Nomura S, Kudo E, HamamotoY. An autopsy case of sarcoidosis in the intestinal canal. Bull Osaka Med School 1972;18:48-55.

2 Clague RB. Sarcoidosis or Crohn's disease? $\mathrm{Br}$ Med $\mathrm{f}$ 1972;3:804.

3 Sprague R, Harper P, McLain S, Trainer T, Beeken W Disseminated gastrointestinal sarcoidosis. Case report and review of the literature. Gastroenterology 1984;87:421-5.

4 Tinker MA, Viswanathan B, Laufer H, Margolis IB. Acute appendicitis and pernicious anemia as complications of gastrointestinal sarcoidosis. Am f Gastroenterol 1984;79:868-72.

5 Bulger K, O'Riordan M, Purdy S, O'Brien J, Lennon J. Gastrointestinal sarcoidosis resembling Crohn's disease. Am $\mathcal{F}$ Gastroenterol 1988;83:1415-7.

6 Rauf A, Davis P, Levendoglu H. Sarcoidosis of the small intestine. Am f Gastroenterol 1988;83:187-9.

7 James DG. Miscellaneous involvement. In: James DG, Williams WJ, eds. Sarcoidosis and other granulomatous disorders. Problems in internal medicine. Vol 24. Philadelphia: disorders. Problems in internal medic

8 Tweedie JH, McCann BG. Metastatic Crohn's disease of thigh and forearm. Gut 1984;25:213-14.

9 Lemann M, Messing B, D'Agay F, Modigliani R. Crohn's disease with respiratory tract involvement. Gut 1987;28:1669-72.

10 Siltzbach LE, Vieria LOBD, Topilsky M, Janowitz HD. Is there Kveim responsiveness in Crohn's disease? Lancet 1971;ii:634-5.

\title{
Immunoperoxidase techniques and histology in the diagnosis of rhabdomyolysis related acute renal failure
}

\author{
P N Cooper, J Tapson, A R Morley
}

\author{
Department of \\ Histopathology, Royal \\ Victoria Infirmary, \\ Newcastle upon Tyne \\ P N Cooper \\ A R Morley \\ Department of Renal \\ Medicine, Freeman \\ Hospital, Newcastle \\ upon Tyne \\ J Tapson \\ Correspondence to: \\ Dr P N Cooper, \\ Department of Pathology, \\ Royal Victoria Infirmary, \\ Queen Victoria Road, \\ Newcastle upon Tyne, \\ NE1 4LP \\ Accepted for publication \\ 24 February 1992
}

\begin{abstract}
A case of rhabdomyolysis associated acute renal failure (RM-ARF) occurring as a result of strenuous exercise is presented. Diagnostic renal biopsy was performed. The histological appearances, combined with immunoperoxidase staining for myoglobin, allowed a positive diagnosis of RM-ARF to be made and excluded the possibility of glomerulonephritis. The patient recovered completely after a stormy clinical course.
\end{abstract}

Rhabdomyolysis can be the result of many diverse factors including direct muscle injury, excessive muscle activity, ischaemia, immunological disease, metabolic disorders, drugs, toxins, infection and genetic disorders. In two large series rhabdomyolysis associated acute renal failure (RM-ARF) represented between
$5 \%$ and $8.6 \%$ of cases of acute renal failure. $^{12}$

In the absence of trauma the clinical symptoms and signs of muscle damage are unreli$\mathrm{able}^{23}$ and diagnosis depends on finding raised serum creatine phosphokinase (CPK) activity and o-toluidine positive urine and golden coloured granular casts on microscopical examination of the urine. The prognosis of RM-ARF is good provided prompt treatment is given. ${ }^{1}$

We report a case of exertion related RMARF in a 17 year old soldier who developed RM-ARF following exercise.

\section{Case report}

A 17 year old private collapsed after running for 7.5 miles wearing army boots and a full backpack on a day in October during which the temperature reached a maximum of $13.9^{\circ} \mathrm{C}$. 


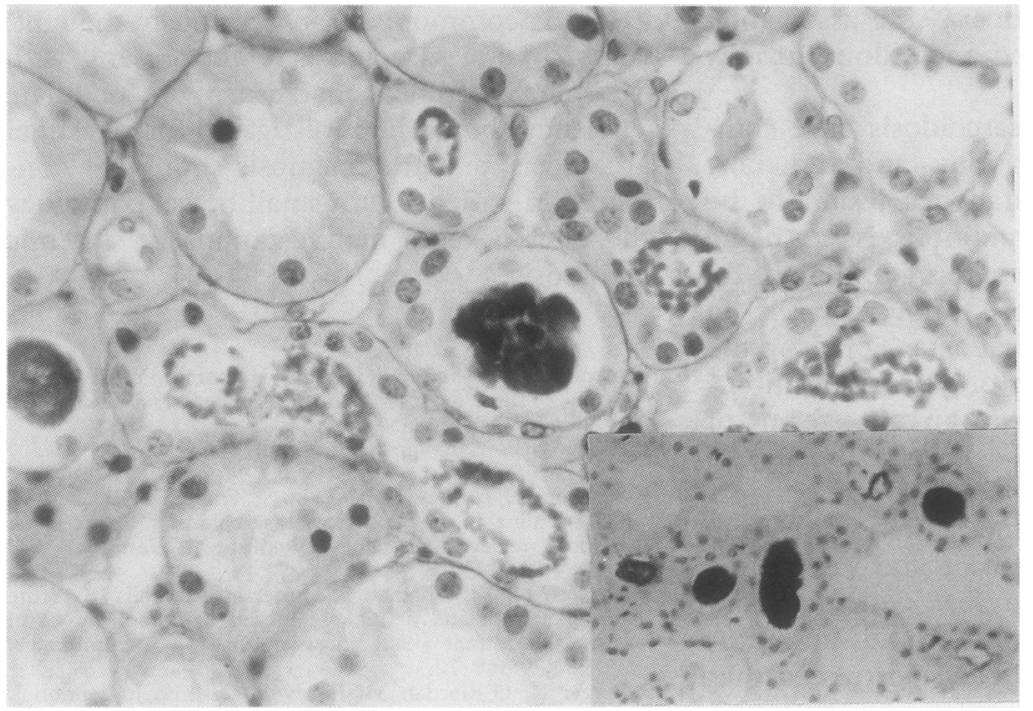

Figure 1 Renal biopsy specimen (haematoxylin and eosin). Inset: immunoperoxidase stain for myoglobin.

He was accustomed to exercise of this severity but had not increased his fluid intake before or during the exercise. On arrival at the local military hospital he complained of generalised abdominal and leg pains. On examination he was clinically dehydrated and had swollen, tender thigh muscles.

The following morning he was noted to be oligoanuric despite adequate rehydration. Investigations showed serum $\mathrm{K}^{+}(7.9 \mathrm{mmol} / \mathrm{l})$; urea $(32 \cdot 2 \mathrm{mmol} / \mathrm{l})$, and creatinine $(566 \mu \mathrm{mol} / \mathrm{l})$. Intravenous insulin and dextrose and rectal calcium resonium were administered to correct the hyperkalaemia. He was transferred to the renal unit, Royal Victoria Infirmary (RVI), Newcastle upon Tyne.

His blood cell count was $21.6 \times 10^{9} / 1$. Urine testing revealed protein ++++ and blood $t+++$. Microscopical examination of the centrifuged urine sediment showed numerous golden coloured granular casts and crenated erythrocytes. Haemodialysis was started.

Although the clinical presentation suggested a diagnosis of acute rhabdomyolysis, confirma-

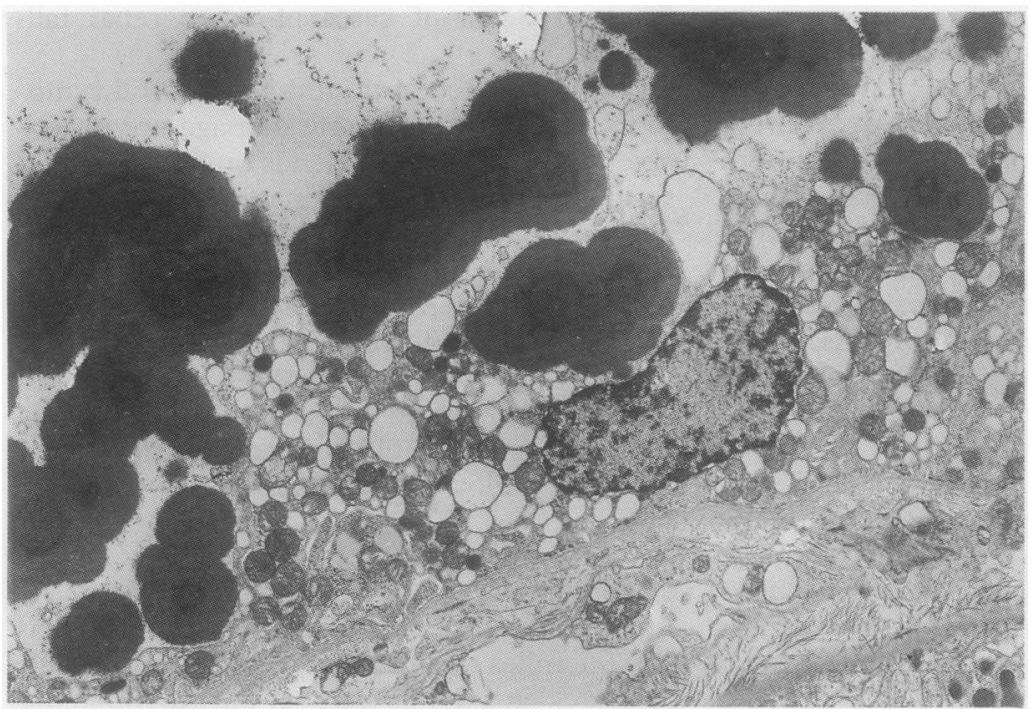

Figure 2 Renal biopsy specimen: electron microscopy. tory biochemical test results were not yet available. Acute oliguric renal failure in the presence of crenated red cells could also have been explained by a rapidly progressive glomerulonephritis. As this condition would require specific treatment a renal biopsy was performed.

Histological analysis showed a total of 45 normal glomeruli. There was no evidence of glomerulonephritis. Most tubules also appeared normal. Some, however, showed dilatation with flattening of epithelial cells. Occasional tubules contained oxalate crystals. Many contained coarsely granular proteinaceous deposits either clumped or forming a ring within the lumina of the tubules (fig 1). The stroma and the vessels present showed no pathology. Immunoperoxidase stains for myoglobin showed strong positivity within the granular tubular casts and also within the cytoplasm of tubular cells (inset fig 1). No positivity was seen on immunofluorescence staining of frozen tissue for IgG, IgM, IgA, C3, $\mathrm{C} 4$ or fibrin. Electron microscopical examination showed vacuolated tubular cells with laminated intratubular casts with a dense inner matrix (fig 2). Fortuitously, a small piece of skeletal muscle from the abdominal wall was included with the renal biopsy specimen. This acted as an internal control for the myoglobin stain but also showed changes of rhabdomyolysis in the form of extensive necrosis of muscle fibres with minimal inflammation.

During the first day of his admission to the RVI transfer to the intensive care unit for sedation and ventilation was necessitated by the development of status epilepticus. Investigations showed serum ionised $\mathrm{Ca}^{++}$of $0.93 \mathrm{mmol} / 1$ (reference range $1.19-1.37 \mathrm{mmol} / \mathrm{l}$ ); phosphate of $2.45 \mathrm{mmol} / 1(0.73-1.33)$; urate of $1.08 \mathrm{mmol} / 1(0.15-0.42)$. At this stage the results of tests taken on admission to the RVI showed a serum lactate dehydrogenase activity of $13500 \mathrm{IU} / 1(<430)$ and a creatinine phosphokinase of $>15000 \mathrm{IU} / 1 \quad(<175)$. Urine myoglobin, interestingly, was negative. A right lower lobe pneumonia, deep vein thrombosis relating to the femoral dialysis catheter, and profound hypercalcaemia with ionised $\mathrm{Ca}^{++}$ peaking at $2.09 \mathrm{mmol} / 1$ complicated his period of intensive care.

\section{Discussion}

Myoglobin released from damaged muscle was implicated in the aetiology of acute renal failure by Bywaters and Beal in $1941 .{ }^{4}$ It is now accepted that rhabdomyolysis and myoglobinuria with or without traumatic skeletal muscle injury is associated with acute renal failure. However, the pathophysiology involved remains controversial. Suggested mechanisms include reduced glomerular filtration rate, tubular obstruction, and back leakage of filtrate across damaged tubular epithelium. ${ }^{1}$

Many of the reported examples of RM-ARF due to physical exercise relate to military personnel. ${ }^{56}$ In 1960 Howenstine reported on the effects of exercise on US Navy recruits. 
Within 24 hours 60 out of 28000 were passing brown urine and 12 were admitted to hospital, two of whom developed acute renal failure. Myoglobinuria was detected in eight of 18 of the recruits tested.

The proportion of cases of acute renal failure associated with rhabdomyolysis is reported in two large series as $5 \%^{1}$ and $8.6 \%$. $^{2}$ The prognosis is good if treatment is instituted promptly ${ }^{2}$ and indeed is reported as being better than that of acute renal failure from other causes. ${ }^{1}$ Prompt diagnosis and treatment are essential because serious cardiotoxic metabolic abnormalities may occur early in the disease. ${ }^{1}$ The diagnosis of RM-ARF relies on clinical observations, biochemical tests on blood and urine, and in problematical cases on renal biopsy.

The clinical history and physical examination are often misleading as many of the patients are incoherent or comatose at presentation. Furthermore, muscle pain is absent in $50 \%$ of patients at admission and detectable muscle swelling is seen in only a small proportion of cases of rhabdomyolysis (four out of 87). ${ }^{3}$ Our patient was mentally alert and complained of muscle discomfort. His thigh muscles were swollen and tender.

The diagnosis of RM-ARF is usually confirmed by biochemical tests on urine and blood. Urinalysis, however, has diagnostic limitations. First it is obviously not possible in the face of total anuria. Secondly the o-toluidine test for myoglobinuria is unreliable. ${ }^{23}$ Thirdly, neither pigmented granular casts in the urine nor o-toluidine positivity are specific to myoglobinuria, both being present in haemoglobinuria as well. ${ }^{2}$

Myoglobin is rapidly cleared from the plasma and thus both urine and serum concentrations may be minimal by the time of admission. In contrast, creatine phosphokinase (CPK) activities decline much more slowly and are thus a more reliable indicator of rhabdomyolysis. ${ }^{3}$ Indeed CPK activities are significantly increased in all patients with RM-ARF. ${ }^{2}$ Thus in the face of an increased CPK activity negative urine screening tests would not exclude the diagnosis of rhabdomyolysis associated acute renal failure.

The availability of biochemical tests limits their usefulness in making an early diagnosis of RM-ARF. In our case the patient was admitted to the RVI on a Sunday. Laboratory services were unable to perform assays for either serum CPK or urinary myoglobin. Support for the clinical diagnosis of RM-ARF rested only on the presence of golden, granular urinary casts and the refractory hyperkalaemia. As the result of a missed early diagnosis of rapidly progressive glomerulonephritis may be irrevocable end-stage renal failure, histological confirmation of the diagnosis of RM-ARF was obtained.

Bywaters and Beal described renal histology in four necropsy cases who sustained severe crush injuries in air-raids during the Second World War. ${ }^{4}$ They described the changes of acute tubular necrosis and the presence of ribbon-like casts in distal tubules. As in our case renal biopsy has been performed in cases of RM-ARF where there was clinical diagnostic difficulty. ${ }^{2-9}$ Histological examination in all these papers is reported as indicating varying degrees of acute tubular necrosis with tubular casts. Electron microscopical examination showed that these comprised granular osmiophilic material. ${ }^{7}$ Kagen was the first to identify this material as myoglobin, using immunofluorescence labelling. ${ }^{10}$ Immunofluorescent material was present in granular casts mainly in distal tubules, loops of Henle, and collecting ducts, particularly closer to the medulla. Tubular cells also showed cytoplasmic positivity. Immunoperoxidase stains for myoglobin were performed on the three renal biopsy specimens obtained from 11 patients reported by Thomas and Ibels in $1985 .^{2}$ The biopsy specimens showed varying degrees of acute tubular necrosis with myoglobin deposition in tubules confirmed by immunoperoxidase. They concluded that immunoperoxidase confirmation of the presence of myoglobin in a renal biopsy specimen is useful in arriving at a precise diagnosis. ${ }^{2}$ Immunoperoxidase stains for myoglobin were also useful in diagnostic cases described by Ellis et al and Saltissi et $a .^{9}$

Renal biopsy specimens may be the quickest reliable method of differentiating glomerulonephritis from RM-ARF in problem cases. Howenstine $^{5}$ found renal biopsy specimens diagnostic in three cases of diagnostic difficulty and Jackson ${ }^{6}$ in one case. In all four cases the differential diagnosis was between glomerulonephritis and tubular damage.

The biopsy specimen in our case showed features similar to those described before (see above). In particular, granular casts, many in the form of ribbons were seen, mostly in distal tubules and collecting ducts.

The use of the immunoperoxidase technique permitted the positive identification of myoglobin within the tubules. This, combined with the absence of histological evidence of glomerulonephritis, allowed the clinical diagnosis of rhabdomyolysis related acute renal failure to be made with confidence and appropriate treatment to be started with a favourable clinical result.

1 Grossman RA, Hamilton RW, Morse BM, Penn AS, Goldberg $M$. Non-traumatic thabdomyolysis and acute renal failure. $N$ Engl f Med 1974;291:807-11.

2 Thomas MAB, Ibels LS. Rhabdomyolysis and acute renal failure. Aust NZ $\mathcal{J}$ Med 1985;15:623-8.

3 Gabow PA, Kaehny WD, Kelleher SP. The spectrum of rhabdomyolysis. Medicine 1982;61:141-52.

4 Bywaters EGL, Beall D. Crush injuries with impairment of renal function. Br Med $\mathcal{f} 1941 ; 1: 427-32$.

5 Howenstine JA. Exertion-induced myoglobinuria and hemoglobinuria. $\mathcal{F A M} A$ 1960;173:493-9.

6 Jackson RC. Exercise induced renal failure and muscle damage. Proc Roy Soc Med 1970;63:566-70.

7 Chagnac A, Rudniki C, Zevin D, Braslavsky D, Zahavi I, Levi J. The morphological changes in acute renal failure due to rhabdomyolysis following viral infection. Nephron 1982;32:75-7.

8 Saltissi D, Parfrey PS, Curtis JR, et al. Rhabdomyolysis and acute renal failure in chronic alcoholics with myopathy, 1984;21:294-300.

9 Ellis CJ, Dewhurst AG, Cooper M, Brenton DP, Dathan JRE. Myoglobinuria - the importance of reaching a firm diagnosis-a patient with defectiv
Postgrad Med $₹$ 1990;66:235-7.

10 Kagen LJ. Immunofluorescent demonstration of myoglobin in the kidney. Am $\mathcal{F}$ Med 1970;48:649-53. 